\title{
Minería de datos, una innovación de los métodos cuantitativos de investigación, en la medición del rendimiento académico universitario
}

\author{
Luis María Dicovskiy Riobóo ${ }^{1}$ \\ Manuel Enrique Pedroza ${ }^{2}$
}

\section{RESUMEN}

En este ensayo sobre calidad universitaria y su medición, se hace una revisión teórica sobre los factores que inciden en el rendimiento académico. En la revisión teórica, se encontró que hay consenso que las mayores deserciones ocurren en el primer año de la universidad y que estas causas están también vinculadas a la historia preuniversitaria del estudiante. La asistencia a clases de forma regular y la motivación a su carrera, también son aspectos importantes que resaltan para mejorar el rendimiento académico. El factor género: varón o mujer, es un aspecto que se debe considerar en cualquier estudio de rendimiento académico. Se discute que las bases de datos académicas que están en los centros universitarios, son un material importante en cualquier investigación académica, y que deberían ser estudiadas por minería de datos, como un método innovador dentro los métodos tradicionales de investigación cuantitativa. La minería de datos permite extraer nuevos conocimientos, a partir de base de datos que surgen de la acumulación de información académica en el tiempo.

Palabras clave: innovación de procesos, minería de datos, rendimiento académico, calidad de la educación universitaria.

Recibido: 25 de julio de 2017

Aceptado: 07 de noviembre de 2017

1 Docente Universidad Nacional de Ingeniería, Sede Regional Norte, Estelí. Estudiante del Programa de Doctorado en “Gestión y Calidad de la Investigación Científica”. UNAN Managua. Correo electrónico: luis.dicovskiy@norte. uni.edu.ni

2 Profesor Titular de la UNAN-Managua. Coordinador del Programa de Doctorado en "Gestión y Calidad de la Investigación Científica”. UNAN Managua. Correo electrónico: hppedroza@gmail.com 


\title{
Mining Data, an innovation of quantitatives research methods, in the measurement of university academic performance
}

\begin{abstract}
In this essay on university quality and its measurement, a theoretical revision is made on the factors that affect the academic performance. In the theoretical review, it was found that there is consensus that major desertions occur in the first year of university and that these causes are also linked to the student's pre-university history. Regular attendance and career motivation are also important aspects that stand out in order to improve academic performance. The gender factor: male or female, is an aspect that should be considered in any study of academic performance. It is argued that the academic databases that are in the university centers, are an important material in any academic research, and that can be studied by data mining. Process that allows to extract new knowledge, data that arise from the accumulation of academic information in the time.
\end{abstract}

Keywords: process innovation, academic performance, university quality, data mining.

\section{INTRODUCCIÓN}

La calidad del Rendimiento Académico no solo impacta en el futuro estudiante y en su familia, sino en la sociedad en general que, al desertar o reprobar, pierde la oportunidad de un profesional formado localmente y con capacidad de incidir en el desarrollo tecnológico de la región. Además de las altas pérdidas económicas que tiene el país, ya que la formación de universitarios es la formación más cara entre los diferentes niveles educativos.

Una manera de disponer información histórica de la conducta de los estudiantes, son las bases de datos académicas con que cuentan las universidades nicaragüenses. Esta información, suele ser completa y cronológica. Otra ventaja es que esta información generalmente se encuentra de forma digital. Allí se genera una oportunidad de estudios multivariados, factoriales, de interacciones con medidas repetidas en el tiempo, uso de modelos lineales generalizados, etc., para tratar de entender las múltiples causas que han favorecido en el éxito o los fracasos académicos. También de estos análisis se pueden proponer recomendaciones educativas que puedan aportar a la mejora en la eficiencia de las formas de graduación de los estudiantes universitarios.

A continuación, se hace una revisión teórica sobre los factores que inciden en el rendimiento académico y de manera particular el factor género. También se discute la forma de medir el rendimiento académico y la incorporación de la minería de datos cómo un proceso innovador, que permite aplicar métodos y técnicas cuantitativas modernas para analizar las bases de datos 
académicos ya existentes. Finalmente se presentan dos investigaciones locales, realizadas en la UNI Sede Regional Norte, donde se aplican principios de la minería de datos.

\section{DESARROLLO}

\section{Factores que inciden en el Rendimiento Académico Universitario}

Para interpretar el rendimiento académico, se debe de considerar que este es un fenómeno multicausal, involucra distintos factores y espacios temporales que intervienen en el proceso de enseñanza-aprendizaje. Entre estas causas coexisten determinantes personales, determinantes sociales y determinantes institucionales. Como: determinantes "personales" se tienen: las competencias cognitivas, motivación, condiciones cognitivas, auto concepto académico, autoeficacia percibida, bienestar psicológico, satisfacción y abandono con respecto a los estudios, asistencia a clases, inteligencia, aptitudes, sexo, formación académica previa a la Universidad, nota de acceso a la universidad. Como determinantes "sociales" están: las diferencias sociales, entorno familiar, nivel educativo de los progenitores o adultos responsables del estudiante, nivel educativo de la madre, contexto socioeconómico, y variables demográficas. Como determinantes institucionales están: elección de los estudios según interés del estudiante, complejidad en los estudios, condiciones institucionales, servicios institucionales de apoyo, ambiente estudiantil, relación estudiante - profesor y pruebas específicas de ingreso a la carrera, (Garbanzo Vargas, 2007, págs. 47-60).

Guido Chávez, (2015, págs. 22-61), en la revisión bibliográfica de su tesis de maestría explica que los factores que inciden en el rendimiento académico se pueden considerar como: objetivos y subjetivos. Los factores subjetivos serían: motivacionales, vocacionales, metodologías de estudio del estudiante, socioeconómicos del entorno, pedagogía y metodología de enseñanza, y las formas de culminación de estudio. En lo que se refiere a factores objetivos estarían: retención estudiantil, deserción, aprobación de asignaturas, aspectos socio productivos del estudiante, rigor científico de las asignaturas del eje de investigación y la eficiencia terminal.

Abordando la deserción académica universitaria y lo complejo de su estudio, Vicent Tinto (1989), concluye que:

"El campo de la investigación del abandono escolar se presenta desordenado, fundamentalmente, porque hemos sido incapaces de convenir los tipos de comportamientos que merecen, en sentido estricto, la denominación de deserción. Como resultado existe confusión y contradicción en lo que se refiere al carácter y a las causas del abandono de la educación superior” (pág. 1).

Un estudio en la Universidad Nacional de Córdoba realizado por Goldenhersch y citado por Merlino y Ayllon, (2016), determinó que, el "primer año de la universidad está fuertemente correlacionado con la mayoría de las variables que muestran la historia preuniversitaria del estudiante, a saber: escuela secundaria, ocupación de los padres, educación de los mismos, sexo, con quién vive y cómo costea sus estudios", fin de cita. 
Edel Navarro, (2003, pág. 14), en una investigación en el Instituto Tecnológico y de Estudios Superiores de Monterrey, Campus Toluca, en México, al comparar el promedio de secundaria y los resultados de sus calificaciones en el primer año de preparatoria, encontró que estas tuvieron un decremento del 4\%, lo que le permitió concluir que "existe la necesidad de brindar alternativas de apoyo y orientación educativa a los alumnos durante ésta fase académica inicial, y por supuesto, a lo largo de su educación preparatoria”. Ruiz, Ruiz, \& Ruiz, (2010, pág. 10), en su estudio con estudiantes de la carrera de Ingeniería Biomédica, Universidad Nacional de Tucumán, Argentina, encontraron que el factor "avance en la carrera" está fuertemente vinculado a la "rapidez" con la que el alumno avanza de año en año, más allá de las notas con que aprueba sus exámenes.

También es importante para mejorar el rendimiento académico, la asistencia a clases, sobre todo en primer año de la carrera. En la universidad de Barcelona se encontró en un estudio, que más del $90 \%$ de los aprobados y notables, corresponden a alumnos que han asistido más del $80 \%$ de las clases. Hallándose una relación matemática entre la asistencia a clases y el de aprendizaje del alumno universitario (Bartual Figueras \& Poblet Farrés, 2009, pág. 179).

La motivación a la carrera en estudio, que esta sea la que se quiere estudiar en primera opción es, además, un factor importante para entender las causas principales de deserción universitaria. Un estudio en la Universidad de Costa Rica, UCR, determinó que los estudiantes que no lograron ingresar a la carrera que desean seguir, eran un 63.4\% de los estudiantes desertores estudiados en esta investigación (Abarca Rodríguez \& Sánchez Vindas, 2005, pág. 14).

Un estudio con estudiantes universitarios en Valencia encontró "correlaciones significativas entre las actitudes hacia el aprendizaje de los estudiantes universitarios y el rendimiento académico, lo que demostraba que se daba una asociación significativa entre actitudes y rendimiento" (Gargallo López, Pérez Pérez, Serra Carbonell, Sánchez i Peris, \& Ros Ros, 2007, pág. 10). Este es un buen ejemplo del uso de métodos y técnicas estadísticas para determinar asociaciones significativas vinculadas al rendimiento académico.

\section{Rendimiento Académico y Género}

Las bases de datos académica, fácilmente pueden particionarse en dos grupos: varón y mujer, esto permite que fácilmente, se pueda tratar de buscar conductas diferenciadas por género. En lo que se refiere a diferencias entre géneros, Vargas M. (2010, pág. 319), en su estudio sobre los factores que determinan el rendimiento académico en matemáticas en la UNI expresa, que "se estima que no existan diferencias significativas entre hombre y mujeres con respecto al rendimiento académico en base de las habilidades intelectuales, (si ocurre) se espera encontrar aspectos sociales propios del contexto que expliquen la conducta diferenciada entre ambos géneros".

Laguna Gámez, (2016, pág. 50) en un artículo de su tesis doctoral, al estudiar los principales factores

que han motivado el abandono de los estudiantes de la carrera de Desarrollo Rural Sostenible, del 
Programa Universidad en el Campo, que ejecuta UNAN FAREM Matagalpa, determinó que el sexo y la edad no fueron variables determinantes en que el abandono se profundizara, fueron variables sin diferencias significativas.

Sin embargo, Giovagnoli, (2002, pág. 24), encontró un comportamiento diferenciado entre géneros con estudiantes universitarios en Rosario, Argentina, cuando estudió los determinantes de la deserción universitaria. El observó, que el género jugó un papel importante en el abandono de la universidad, un varón tenía una 1.36 veces más posibilidad de abandono que el de una mujer.

Estudiando la percepción del género relacionada con la eficacia de la enseñanza de sus docentes. A estudiantes de la escuela de negocios de la universidad de Dakota del Sur, se les pasaron 381 encuestas donde se analizaron 35 aspectos cualitativos. En 29 de los 35 aspectos, la respuesta media de las mujeres fue mayor que la media de los estudiantes varones, y para 15 de los 29, las diferencias fueron estadísticamente significativas al nivel del 5\%. Los 15 aspectos de la enseñanza que las mujeres clasificaron estadísticamente más alto que los hombres fueron: profesionalismo, retroalimentación oportuna, preparación de clase, organización de las presentaciones, atención, vestimenta profesional, estándares académicos altos, accesibilidad fuera de clase, respeto, entusiasmo, explicaciones alentadoras, ser justos y generación de atracción. (Lavin, Korte, \& Davies, 2012, págs. 4-7).

Al estudiar las percepciones diferenciadas por género también hay que considerar el contexto de las ingenierías y el género, dice Preciado Cortés, Kral, \& Álvarez Ramón, (2015, pág. 39), que "la ingeniería es un área que históricamente ha sido ocupada por hombres; en este espacio la presencia femenina es entendida como un caso excepcional". Esto observación hace suponer que las mujeres que estudian ingeniería, no tienen una conducta promedio de su género.

\section{Rendimiento académico y su medición}

En general, en los centros escolares se acumulan un volumen considerable de datos académicos que son poco aprovechados, y ahí hay un campo muy útil para los profesionales de la educación donde se podrán hacer estudios interesantes para la propia institución y para otros profesionales (Morales Vallejos, 2002, pág. 15).

Garbanzo Vargas, (2007, pág. 43), vincula los conceptos de rendimiento académico y calidad educativa cuando dice que, "el rendimiento académico del estudiantado universitario constituye un factor imprescindible en el abordaje del tema de la calidad de la educación superior, debido a que es un indicador que permite una aproximación a la realidad educativa".

Esto conlleva a afirmar que una de las dimensiones más importantes en el proceso de enseñanzaaprendizaje la constituye el "rendimiento académico del alumno". Tal como lo destacan (Ruiz, Ruiz, \& Ruiz, 2010, pág. 1), “el rendimiento académico de un estudiante universitario es la 
resultante de una multiplicidad de factores, que van desde los personales, hasta los dependientes de la institución y sus docentes", fin de cita.

Dentro de los datos académicos, están los resultados de las evaluaciones. Estas tienen un contenido social, ya que permiten, dentro de unos límites, saber que quienes van a ejercer la profesión dominan los conocimientos, tienen las habilidades y disponen de las actitudes que permiten asegurar que el ejercicio de la práctica profesional será bien realizado (Santos Guerra, 2014, pág. 12).

Si bien el rendimiento del alumno debería ser entendido o explicado a partir de sus procesos de evaluación; la simple medición y/o evaluación de los rendimientos alcanzados por los alumnos no provee por sí misma todas las pautas necesarias para la acción destinada al mejoramiento de la calidad educativa. Unas de las variables más empleadas o consideradas por los docentes e investigadores para aproximarse al rendimiento académico son: las calificaciones. (Navarro, 2003).

Sin embargo, las calificaciones, como medida de los resultados de enseñanza, son producto de diferentes condicionantes, tanto de tipo personal del estudiante, como didácticas del docente, contextuales e institucionales, y que todos estos factores median el resultado académico final (Garbanzo Vargas, 2007, pág. 48) .

Para medir el rendimiento académico, se han definido diferentes índices numéricos, un ejemplo de estos son los Índices de Proceso, de Producto y de Aprobación. Por ejemplo, un índice de Proceso es aquel que considera la cantidad de alumnos promocionados en un curso dentro de un año. Un Índice de Producto es aquel que toma en cuenta la cantidad de alumnos que aprueban el examen final y un Índice de Aprobación es aquel que expresa la cantidad de alumnos aprobados en un año (Ruiz, Ruiz, \& Ruiz, 2010, pág. 1) .

Cuando se calculan índices numéricos hay que tomar en cuenta que cualquiera sea la metodología a emplear en el seguimiento de un alumno, de una cohorte o de toda la población estudiantil, es necesario obtener datos numéricos como: número de ingresantes, promedio de calificaciones, número de materias aprobadas, etc. Estos datos deben ser confiables, repetibles y fácilmente verificables. Sin embargo, además de los indicadores numéricos, un buen seguimiento debiera estar complementado con datos obtenidos de encuestas, entrevistas o talleres de discusión en los que intervengan todos los participantes del proceso enseñanza aprendizaje (Ruiz, Ruiz, \& Odstrcil, 2007, págs. 1-8).

\section{Minería de Datos académicos, una innovación en el análisis datos}

El concepto de innovación de procesos educativos para mejorar la calidad de la educación universitaria, puede ser interpretada de diversas maneras, pero desde una perspectiva funcional se considera que una innovación es la incorporación de una idea novedosa, práctica dentro de 
un conjunto, con la convicción de que el todo cambiará, a partir de las partes que lo constituyen (Salinas, 2004, pág. 2). La Minería de Datos, es sin duda, un método innovador para determinar patrones y modelos en la investigación educativa.

El uso masivo de la computación permitió que surgiera un proceso de investigación nuevo, la Minería de Datos. La cual se basa en el uso de algoritmos computacionales que permiten extraer nuevos conocimientos, de grandes bases de datos que surgen de la acumulación de información que se generan de las actividades cotidianas de las organizaciones. Este conocimiento permite entre otros, conocer anomalías no esperadas y tomar decisiones sobre nuevas situaciones generadas (Martínez Luna, 2011, pág. 53).

La minería o exploración de datos, conocida también como "data mining", se puede definir como: un proceso de descubrimiento de nuevas y significativas relaciones, patrones y tendencias al analizar grandes volúmenes de datos. Este proceso se utiliza hoy en diferentes campos de la ciencia, incluidos aplicaciones financieras, análisis de mercados y comercio, seguros, educación, etc. (Perez Lopez \& Santín González, 2007, pág. 1).

Cuando se analizan bases de datos académicos, se pueden utilizar herramientas estadísticas propias de la minería de datos. La minería de datos educativos, es una disciplina emergente que se centra en la aplicación de datos, herramientas y técnicas de minería a datos relacionados con la educación. Esta se centra en analizar los datos educativos para desarrollar modelos que mejoren las experiencias de aprendizaje y la eficacia institucional (Huebner, 2013, pág. 1).

La Sociedad Internacional de Minería de Datos Educativos, define la minería de datos educativos como "una disciplina emergente, relacionada con el desarrollo de métodos para explorar los tipos únicos de datos que vienen de los entornos educativos, y el uso de esos métodos para comprender mejor a los estudiantes y los entornos en los que aprenden". Dentro de las categorías que se usa en la minería de datos están: Predicción por Clasificación, Regresión, Estimación de la densidad, Clustering o Agrupamientos. Minería de Relaciones por: Reglas de Asociación, Correlación, Extracción secuencial de patrones y Extracción de Datos Causales. Destilación de datos por el juicio humano. Y Descubrimiento con Modelos. (Baker \& Yacef, 2009, págs. 4-5).

Cómo antecedente del uso de la minería de datos para obtener información de conductas ocurridas con los estudiantes de la UNI, Universidad Nacional de Ingeniería, Sede Regional Norte, se pueden citar la experiencia de Montenegro, López y Fonseca, (2011), cuando utilizaron el análisis estadístico Determinante para clasificar potenciales estudiantes exitosos y desertores. Dicovskiy (2013), encontró conductas diferenciadas según el tipo de formación de los docentes. Estos ejemplos se explican a continuación

Buscando un modelo predictivo de deserción y éxito. A partir del historial de notas de 40 estudiantes de la UNI Norte, de Estelí de la carrera de Ing. Agroindustrial, con la técnica de análisis multivariado 
discriminante, se pudo construir un modelo probabilístico que, a partir de las notas obtenidas en el primer semestre de clases, permitiera clasificar los estudiantes en tres categorías: Desertor, De Riesgo y Exitoso. Estas categorías se definieron como: Desertor, aquel estudiante que al cabo de 5 años no había inscrito ninguna asignatura del último año de su carrera; De Riesgo, aquel estudiante que había inscrito 3 o 2 asignaturas de quinto año; y Exitoso, aquel estudiante que había inscrito 4 o más asignaturas de su carrera de quinto año. Este modelo permitió una clasificación correcta de 8 de cada 10 estudiantes desertores y exitosos. Solo con las notas del primer semestre de clases, el modelo predijo con más de un 80 de éxito quienes serían exitosos y quienes no (Montenegro, López, \& Fonseca, 2011, págs. 87-92)

Estudiándose el comportamiento académico de los estudiantes de agroindustria de la UNI Sede regional Norte en el periodo 2005-2012, en función de la formación de sus profesores se separaron dos grupos, aquellas con formación en ciencias de la educación y sin esta, en su mayoría ingenieros. Se determinó que en ambos grupos un comportamiento semejante en el tiempo, en cuanto a "promedio de notas" y "proporción de aprobados", pero si se observó diferencias significativas en las proporciones de estudiantes con notas altas o bajas. Los profesores que tenían una formación previa en ciencias de la educación podían discriminar mejor en sus evaluaciones las diferencias entre los estudiantes. Estos discriminaban mejor entre estudiantes por arriba del promedio y por debajo del mismo al momento de evaluar. Mientras que los profesores empíricos tenían una tendencia a evaluar cerca del promedio, en una banda más estrecha de calificación (Dicovskiy, 2013, pág. 20).

\section{CONCLUSIONES}

Los factores que inciden en el rendimiento académico son multicausales donde coexisten diferentes causas determinantes. Estas causas pueden ser objetivas o subjetivas. Todos estos aspectos inciden para que la interpretación de la deserción o abandono de la educación superior, sea un aspecto complejo de abordar. Sin embargo, hay consenso que las mayores deserciones ocurren en el primer año de la universidad y que estas causas están también vinculadas a la historia preuniversitaria del estudiante. La asistencia a clases de forma regular y la motivación a su carrera también son aspectos importantes que resaltan para mejorar el rendimiento académico.

El género, varón o mujer, es un aspecto que se debe considerar en cualquier estudio de rendimiento académico. También inciden circunstancias particulares del grupo de estudiantes a investigar, por ejemplo, tipo de carrera, puede lograr que se encuentren diferencias en el comportamiento de estas dos categorías.

Para estudiar las causas que afectan el rendimiento académico se propone usar la minería de datos a partir de bases de datos de mediciones evaluativas, que tienen las instituciones educativas y que generalmente se aprovechan poco. La minería de datos es un método innovador, que facilita las herramientas para extraer nuevos conocimientos, de las grandes bases de datos que surgen de la 
acumulación de información académica en el tiempo. De manera local, se han encontrado dos investigaciones académicas que han aplicado exitosamente los principios de la minería de datos.

\section{BIBLIOGRAFÍA}

Abarca Rodríguez,A., \& SánchezVindas,A.(2005). La deserción estudiantilenla educación superior: el caso de la Universidad de Costa Rica. Revista Electrónica "Actualidades Investigativas en Educación”, 5, 1-22. Obtenido de http://www.redalyc.org/pdf/447/44759911.pdf

Baker, R., \& Yacef, K. (2009). The state of educational data mining in 2009: A review and future visions. JEDM-Journal of Educational Data Mining, 1(1), 3-17.

Bartual Figueras, T., \& Poblet Farrés, y. M. (2009). Determinantes del rendimiento académico en estudiantes universitarios de primer año de Economía. Revista de Formación e Innovación Educativa Universitaria, 2(3), 172-181. Obtenido de http://refiedu.webs.uvigo.es/Refiedu/ Vol2_3/REFIEDU_2_3_6.pdf

Dicovskiy, L. M. (2013). Evolución Académica de la carrera de Ingeniería Agroindustrial, UNI Sede Regional Norte, en el periodo 2005-2012. El Higo, 3(1), 18-20.

Garbanzo Vargas, G. M. (2007). Factores asociados al rendimiento académico en estudiantes universitarios, una reflexión desde la calidad de la Educación Superior Pública. Revista Educación, 3(1), 43-63.

Gargallo López, B., Pérez Pérez, C., Serra Carbonell, B., Sánchez i Peris, F., \& Ros Ros, I. (2007). Actitudes ante el aprendizaje y rendimiento académico en los estudiantes universitarios. (1. C. Organización de Estados Iberoamericanos para la Educación, Ed.) Revista Iberoamericana de Educación(42), 1-25.

Huebner, R. (Abril de 2013). A survey of educational data-mining research. Research in Higher Education Journal, 19, 1-13. Recuperado el 5 de Abril de 2017, de https:/www.aabri.com/ manuscripts/121328.pdf

Lavin, A., Korte, L., \& Davies, T. (Diciembre de 2012). Student gender and perceptions of teaching effectiveness 1. Research in Higher Education Journal, 18, 1-16.

Martínez Luna, G. L. (Octubre-Diciembre de 2011). La Minería de Datos. Cómo hallar una aguja en un pajar. Ingenierías, XIV(53), 53-63.

Merlino, A., \& Ayllon, S. (2016). Experiencias en Investigación Educativa. Córdoba, Argentina: Brujas.

Montenegro, J., López, A., \& Fonseca, E. (2011). Automatización de un Sistema Predictivo de Deserción Estudiantil en la UNI Norte, integrado al SIRA. Estelí: UNI Norte.

Morales Vallejos, P. (15 de Enero de 2002). La evaluación académica: conceptos y planteamientos básicos. Cuaderno Monográficos del ICE. Serie Didáctica Número 2, 52.

Navarro, R. (2003). El rendimiento académico: concepto, investigación y desarrollo. REICE Revista Electrónica Iberoamericana sobre Calidad, Eficacia y Cambio en Educación, 1 (2), 1-15. Obtenido de http://www.ice.deusto.es/rinace/reice/vol1n2/Edel.pdf

Perez Lopez, C., \& Santín González, D. (2007). Minería de datos: técnicas y herramientas. Madrid: Paraninfo. 
Ruiz, E., Ruiz, G., \& Odstrcil, M. (25 de Marzo de 2007). Metodología para realizar el seguimiento académico de alumnos universitarios. (OEI, Ed.) Revista Iberoamericana de Educación(42), $1-9$.

Ruiz, G., Ruiz, J., \& Ruiz, E. (25 de Abril de 2010). Indicador global de rendimiento. (OEI, Ed.) Revista Iberoamericana de Educación(52), 1-11.

Salinas, J. (Noviembre de 2004). Innovación docente y uso de las TIC en la enseñanza universitaria. Universidad y Sociedad del Conocimiento, 1(1), 1-16.

Santos Guerra, M. Á. (2014). La Evaluación como Aprendizaje. Cuando la flecha impacta la diana (Segunda ed.). Madrid, España: Narcea, S. A.

Tinto, V. (1989). Definir la Deserción, una cuestión de perspectiva. Recuperado el 30 de Agosto de 2016, de Publicaciones ANUIES: http://publicaciones.anuies.mx/pdfs/revista/Revista71_ S1A3ES.pdf 